\title{
Cancellation Techniques for Co-channel Interference in MIMO- OFDM Systems and Evaluating Their Performance
}

\author{
Varshini Rajesh ${ }^{1}$, A. R. Abdul Rajak ${ }^{1 *}$ \\ ${ }^{1}$ Department of Electrical and Electronics Engineering, Birla Institute of Technology and Science Pilani, Dubai, UAE
}

\begin{abstract}
In a wireless communication system, the transmitted signal is exposed to various surfaces where it bounces and results in several delayed versions of the same signal at the receiver end. The delayed signals are in the form of electromagnetic waves that are diffracted and reflected from the various object surfaces. These result in co-channel interferences for wireless systems. MIMO has proven to be a striking solution for the new generation of wireless systems. MIMO-OFDM system with QPSK modulation is considered as the wireless system for studying the performance of interference cancellation techniques. The BER performance is studied in channels such as Rayleigh and Rician Fading Channels. The effects of interference are reduced to a certain extent by the inclusion of CDMA (spread spectrum technique) as Technique 1 . The effects of interference on this system have been further reduced using the LMS filter as Technique 2. Hence, to show better performance in MIMO-OFDM systems, it is recommended to employ both CDMA and LMS filters to decrease the effects of co-channel interference. It is observed that the parameter BER reduces as the SNR increases for both these channels.
\end{abstract}

\section{Keywords:}

MIMO-OFDM;

Wireless System;

Interference Mitigation;

CDMA-LMS.

\section{Article History:}

Received: 18 August 2021

Revised: 28 October 2021

Accepted: $\quad 08 \quad$ November 2021

Published: $01 \quad$ December 2021

\section{1- Introduction}

Broadband technologies like WiMax and Wireless LAN employ multi-carrier modulation techniques like OFDM. OFDM is a multiplexing technique that uses the concept of orthogonality that transmits signals digitally on multiple carrier frequencies. It multiplexes or divides a high-speed stream of data and places them on low-speed narrow-spaced subcarriers. This in turn makes the data less susceptible to frequency selective fading, inter-channel interference (ICI), and inter-symbol interference (ISI). It has several applications in a range of cellular systems, including the latest 5G technology. The necessity of this project is that the performance of MIMO system needs to be improved by reducing the effect of interference. The introduction of CDMA led to a reduction in co-channel interference, and employing LMS filter in CDMA-MIMO-OFDM systems is the innovation implemented in this project. The organization of writing this article is as follows. Section 1 deals with OFDM and Section 2 explains about MIMO systems. Section 3 describes interference and Section 4 elucidates the literature survey used. The Problem statement of article is revealed in section 5. Section 6 and Section 7 exhibit solutions and results for the problems identified. Section 8 finally discusses the results obtained

\section{1-1-OFDM Systems}

OFDM is a multi-carrier transceiver scheme. Since several subcarriers are involved in this technique and to separate these, a guard interval or guard band is needed to combat Adjacent Channel Interference (ACI). The sub-channels at

*CONTACT: abdulrazak@dubai.bits-pilani.ac.in

DOI: http://dx.doi.org/10.28991/esj-2021-01313

(C) 2021 by the authors. Licensee ESJ, Italy. This is an open access article under the terms and conditions of the Creative Commons Attribution (CC-BY) license (https://creativecommons.org/licenses/by/4.0/). 
these frequencies follow orthogonality as shown in Equation 1 given below.

$\frac{1}{N} \sum_{n=0}^{N-1} e^{j 2 \pi \frac{m}{T_{s y m}} n T_{S}} e^{-j 2 \pi \frac{i}{T_{s y m}} \cdot n T_{S}}=\left\{\begin{array}{l}1, \forall \text { integers } \mathrm{m}=\mathrm{i} \\ 0, \quad \text { otherwise }\end{array}\right.$

Here, time-limited complex signals, are represented by $\left\{e^{j 2 \pi f_{m} t}\right\}_{m=0}^{N-1}$. That is nothing but the different subcarriers in an OFDM signal at a frequency $f_{m}=m / T_{\text {sym }}$, where $0 \leq t \leq T_{\text {sym }}$. The integral of the product of these signals for their fundamental period is zero due to orthogonality. However, this work considers discrete samples, so they are taken at various sampling periods $t=\frac{n T_{s y m}}{N}=n T_{s}, n=0,1,2, \ldots, N-1$. Thus, to be free of ICI, an OFDM signal has to comprise the orthogonality property. Figure 1 represents the block diagram for OFDM systems.

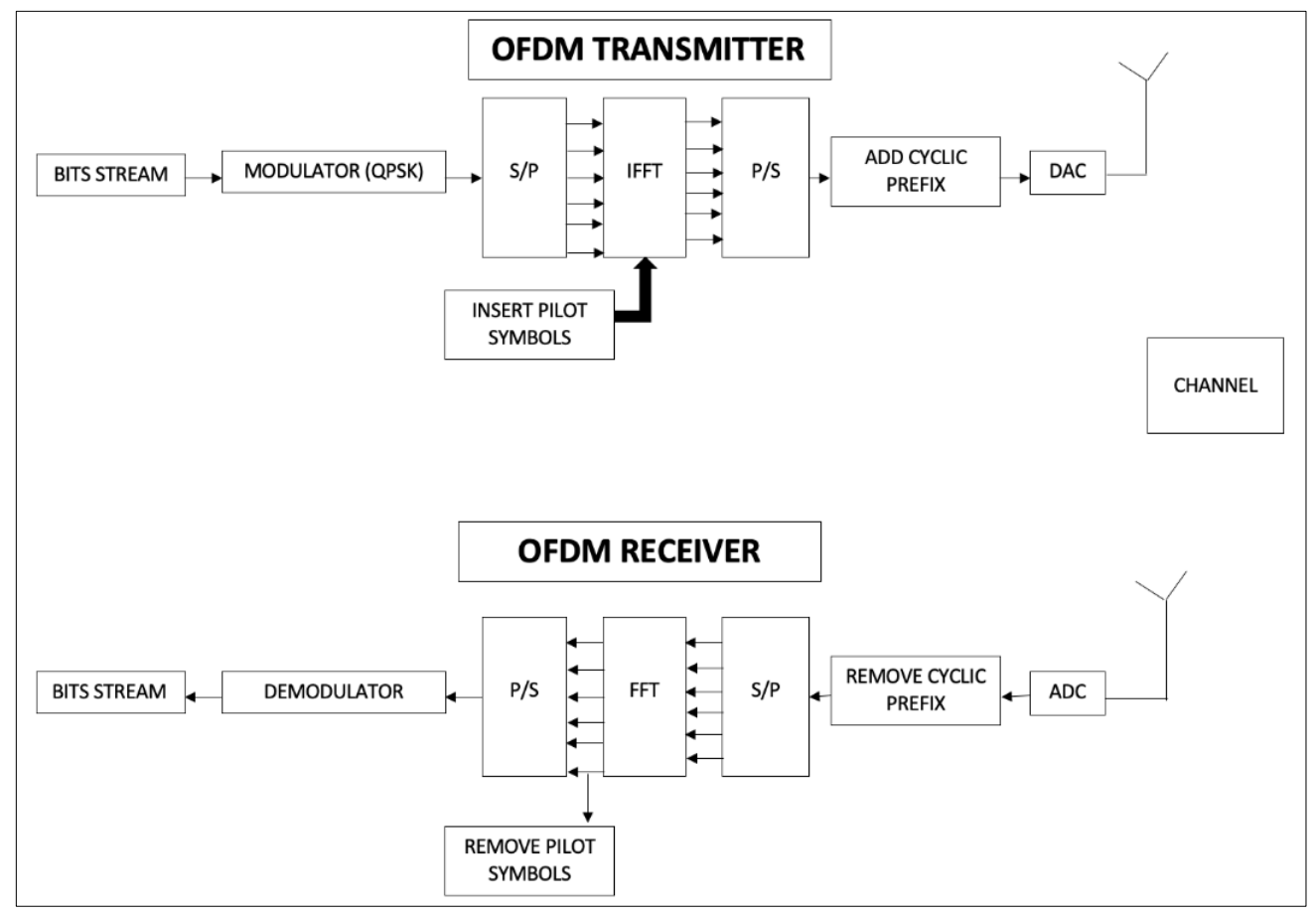

Figure 1. Complete transceiver model of an OFDM system.

\section{1-2-Transmitter Side}

In Figure 1, the sequence of bits enters the modulation block and undergoes QPSK modulation resulting in a complex output symbol. These symbols are converted into a parallel block by using a Serial-to-parallel (S/P) converter. The function of introducing Pilot symbols is to reduce interference and BER to a certain extent. Symbol mapping is done after the insertion of pilots using an Inverse Fast Fourier Transform (IFFT) block. This IFFT block converts the symbols in the frequency domain to the time domain. Equation 2 [1] depicts the output of the IFFT block.

$x(n)=\frac{1}{n} \sum_{m=0}^{N-1} X_{m} e^{\frac{2 j \pi m n}{N}}, 0<n<N-1$.

Here, $\mathrm{x}(\mathrm{n})$ represents the corresponding completely discrete-time OFDM symbols and $\mathrm{X}_{\mathrm{m}}$ is each symbol in the frequency domain. The parallel block of symbols is converted into a serial line of digits with the help of a Parallel-toserial (P/S) converter. A copy of the preceding symbols makes cyclic prefix extensions such that there is a guard band interval for the symbols.

\section{1-3-Receiver Side}

The operations performed on the transmitter side are ultimately reversed and their inverses are executed in the receiver implementation. The serial digital bits are converted into a parallel block after the removal of the cyclic prefix. Furthermore, the Fast Fourier Transform (FFT) is performed. Parallel data transmission combined with Frequency Division Multiplexing (FDM) and FFT is to realize OFDM. Equation 3 [2] defines the demodulation process taking place;

$Y[m]=\sum_{n=0}^{N-1} y[n] e^{-j 2 \pi \frac{m n}{N}}=X[m]$

where, $y[n]=\frac{1}{N} \sum_{i=0}^{N-1} X[i] e^{j 2 \pi \frac{i n}{N}} \cdot y[n]$ represents an N-point DFT. These received symbols in Equation 3 are converted back to the frequency domain. 
An OFDM system can reduce Adjacent Channel interference but it does not reduce Co-channel Interference. This OFDM system employs QPSK modulation and the probability of bit error rate is represented by $P_{b}$ and can be derived by the formula shown below in Equation 4 [3].

$P_{b}=\int_{0}^{3 \pi / 4}\left[\operatorname{det}\left(\frac{\sum \sin ^{2}\left(\frac{\pi}{4}\right)}{\sin ^{2}(\theta)}+I\right]^{-1} d \theta\right.$

The theoretical BER for a QPSK model in terms of the Q-function in an AWGN channel can be given as follows in Equation 5.

$P_{b}=Q\left(\sqrt{\left(\frac{2 E_{b}}{N_{0}}\right)}\right)$

where $E_{b}$ is Energy per bit and $N_{0}$ is noise spectral density. Attenuation and phase rotation [4] in a signal modulated by the M-PSK technique can be described as follows in Equation 6.

$S(t)=\sqrt{\left(\frac{2 E_{b}}{T}\right) \cos \left(2 \pi f_{c} t+\frac{(2 m-1) \pi}{4}\right)} 10 \frac{-D n k}{20} e^{\frac{2 j \pi \beta D n}{\lambda}}$

where, $\mathrm{m}=1,2,3 \ldots \mathrm{M}$, is the constellation number for a particular M-phase shift keying technique, $\beta$ is the phase constant and $D$ is delay in each slot. Here, we take $M=4$. $\lambda$ represents wavelength and the $E_{b}$ denotes the energy per bit. The number of slots is given by $n$ [5].

\section{2- MIMO Wireless Systems}

Technological advancements and applications have given rise to an upward motion in the demand for high-speed data and enhanced quality in communication. This causes a depletion in the availability of bandwidth required for speedy and faultless communication systems [6]. Hence, spectrum efficiency became an extremely important factor while designing any wireless communication device. This gives birth to MIMO systems. The significant role played by MIMO systems in communication can be effective in offering high capacity and better quality of transmission. MIMO is an accurate representation of the users in the real world by increasing the number of antennas that could suffice the number of users in a particular sector,

\section{2-1- Multiple Antenna Procedures}

A MIMO system employs an array of antennas. Antennas that transmit electromagnetic waves hop on various obstacles around the environment. Thus, these waves establish multipath and create various delays. This can be defined as multi-path delay, which causes co-channel interference, as seen in Figure 2. It is needed to reduce multipath fading. STBC (Space Time Block Coding) is employed in this system to reduce the multipath problem.

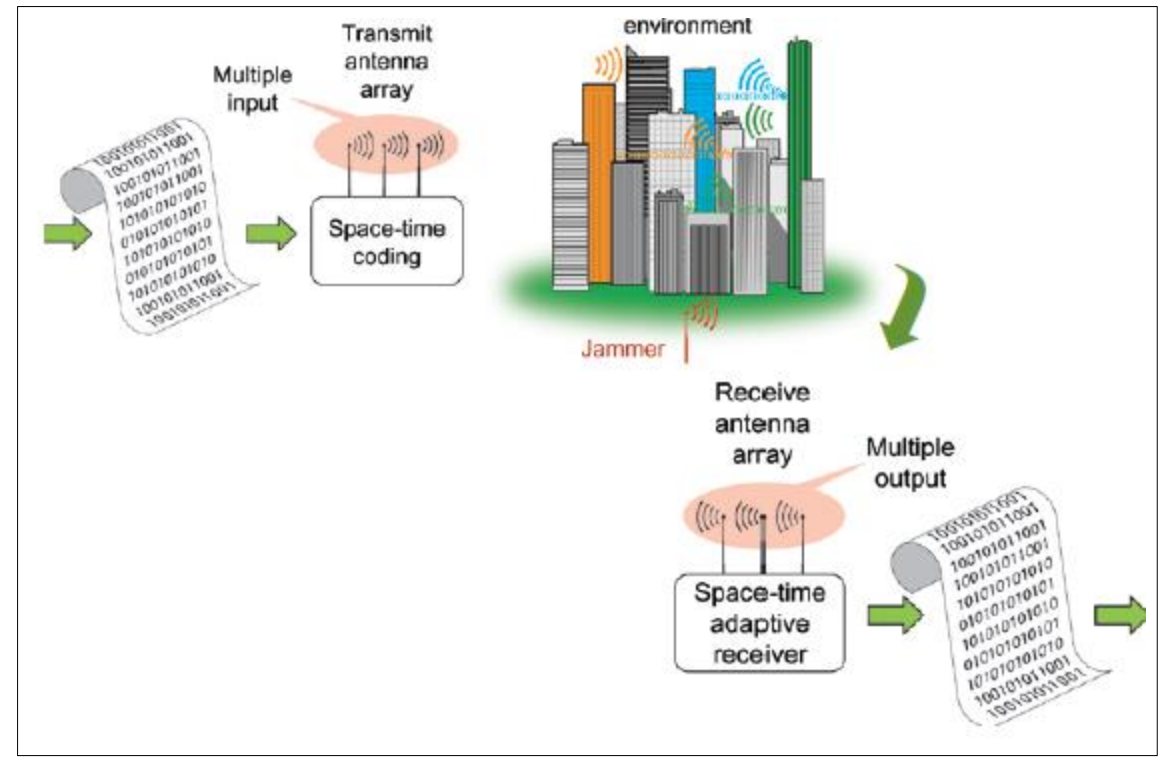

Figure 2. Environment with Multipath [7].

Figure 3a exhibits that signals are being transmitted and received at all possible antenna combinations. The multiple antennas present allow different signals to be received at different time slots at different antennas. 


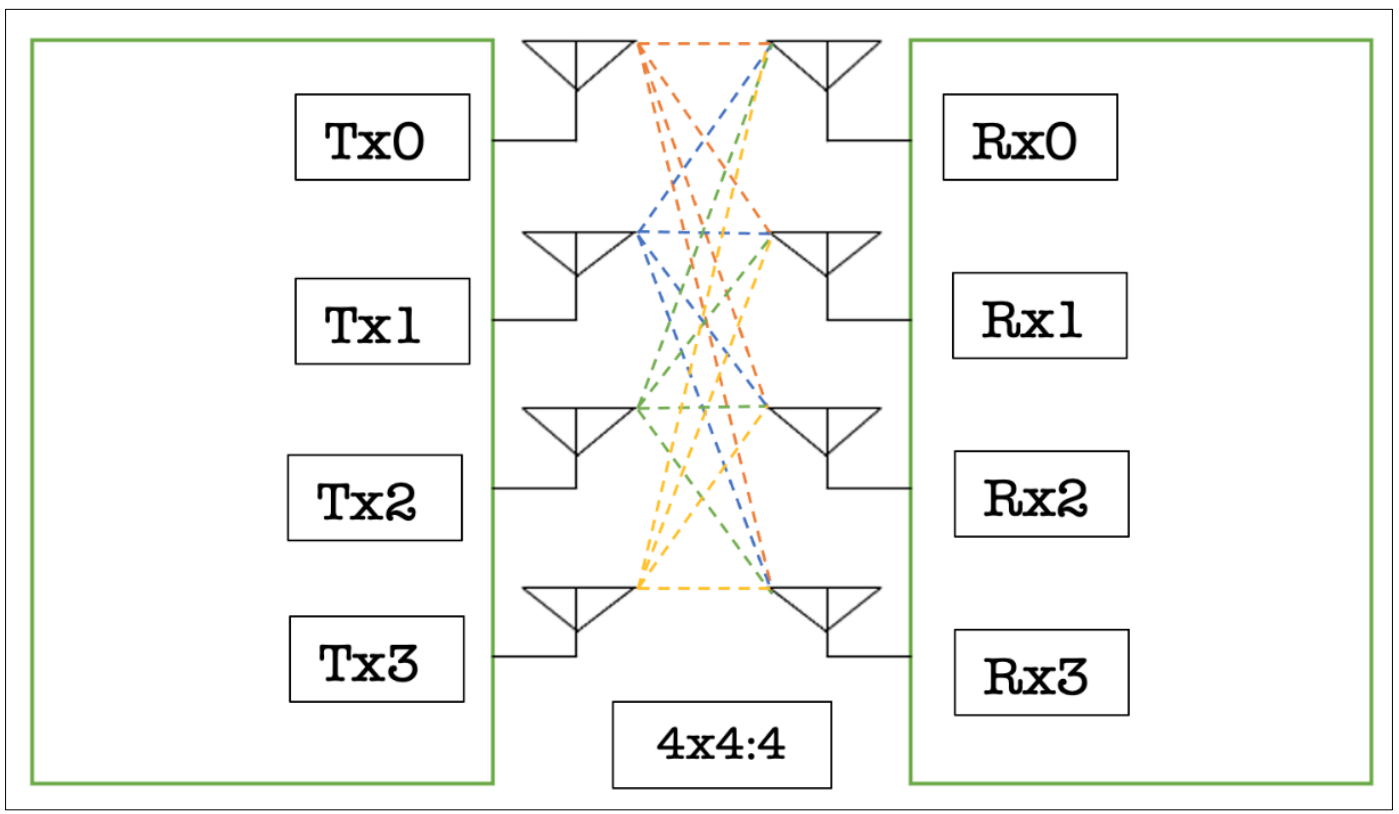

Figure 3. a) MIMO Design.

A MIMO system with $\mathrm{T}$ transmitting antennas and $\mathrm{R}$ receiving antennas are taken in to account. The $j^{\text {th }}$ antenna receives the signal as shown in Equation 7.

$Y_{j}=\sum_{k=1}^{R} h_{j k} x_{k}+r_{k}$

$h=\left[\begin{array}{cccc}\mathrm{h}_{11} & \mathrm{~h}_{12} & \ldots & \mathrm{h}_{1 T} \\ \mathrm{~h}_{21} & \mathrm{~h}_{22} & \cdots & \mathrm{h}_{2 T} \\ \vdots & \vdots & \ddots & \vdots \\ \mathrm{h}_{\mathrm{R} 1} & \mathrm{~h}_{\mathrm{R} 2} & \cdots & \mathrm{h}_{R T}\end{array}\right]$

$r=\left[\begin{array}{c}\mathrm{r}_{1} \\ \mathrm{r}_{2} \\ \vdots \\ \mathrm{r}_{\mathrm{R}}\end{array}\right]$

$y=\left[\begin{array}{c}y_{1} \\ y_{2} \\ \vdots \\ y_{R}\end{array}\right]$

$x=\left[\begin{array}{c}\mathrm{x}_{1} \\ \mathrm{x}_{2} \\ \vdots \\ \mathrm{x}_{\mathrm{R}}\end{array}\right]$

where, $\mathrm{j}=1,2,3 \ldots \mathrm{R}$, the fading factor corresponding to the respective transmit and receive antennas $\mathrm{j}$ and $\mathrm{k}$ is represented by $h_{j k} . r_{k}$ is the noise with respect to receiving antenna k. Equations 8 to 10 represent the matrix representation of the MIMO system and its components are used in Equation $7[8,9]$.

\section{2-2- Space-Time Block Coding (STBC)}

STBC is known to reduce multipath interference in multiple antennas. This technique gets redundant data and computes the weighted average of the same. It combines all the versions of a particular modulated signal. Multiple copies of the received signal are being combined in an optimal way such that maximum information is being extracted. STBC is represented as the matrix given in Equation 12;

$\left[\begin{array}{cccc}\mathrm{s}_{11} & \mathrm{~s}_{12} & \ldots & \mathrm{s}_{1 n} \\ \mathrm{~s}_{21} & \mathrm{~s}_{22} & \cdots & \mathrm{s}_{2 n} \\ \vdots & \vdots & \ddots & \vdots \\ \mathrm{s}_{\mathrm{T} 1} & \mathrm{~s}_{\mathrm{T} 2} & \cdots & \mathrm{s}_{T n}\end{array}\right]$ 
Each row in the matrix of Equation 18 denotes one time slot and each column is the transmission antenna. Going along with a common notation, $s_{i j}$ can be understood as a modulated symbol in time slot ' $\mathrm{i}$ ' from antenna ' $\mathrm{j}$ '. Thus, this matrix helps in combining copies and encoding as per the STBC technique.

\section{2-3- Reference System for Research}

The reference system consists of MIMO with OFDM system as shown in Figure 3.b is implemented in MATLAB Simulink. The channels considered for studies are Rayleigh channel and Rician channel.

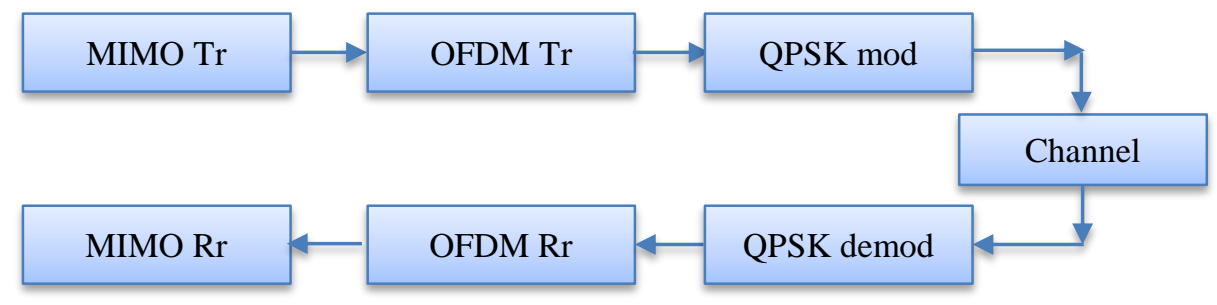

Figure 3. b) Reference system.

\section{3- Interference}

Interference refers to an external aspect that modifies or disrupts a signal when it travels from the transmitter to the receiver. Interference can be due to external noise addition or maybe due to adjacent and co-channel frequencies interfering with the message signal. It can be a major issue since it reduces the strength of a signal at the receiver. This paper concentrates only on Co-channel interference.

\section{3-1- Co-channel Interference}

Figure 4a shows the co-channel model in the channel. The delay blocks $\mathrm{z}^{-1}, \mathrm{z}^{-2}, \mathrm{z}^{-3}$ are added to the original signal using the Sum Block. The strength of these delay blocks is increased with the help of gain blocks. These delay blocks interfere with the transmitted signal in the same channel and hence, it is being termed as co-channel interference.

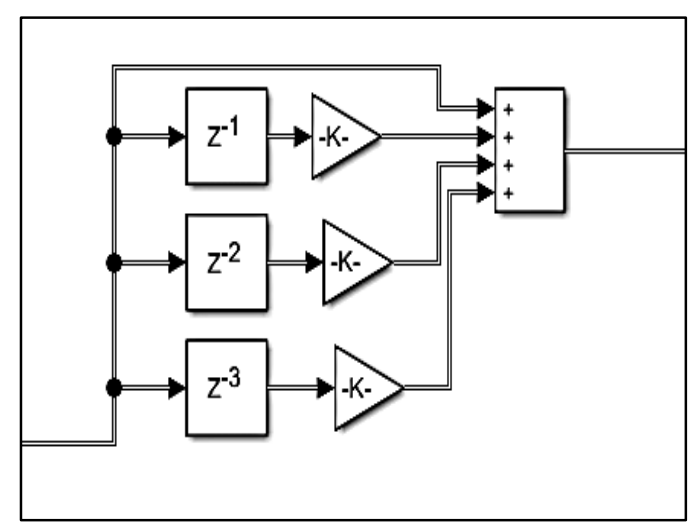

Figure 4. a) Interference caused by the delay blocks.

\section{4- Literature Survey}

Larashati et al. [10] researched Massive MIMO technology with 64 x 64 antennas at $60 \mathrm{GHz}$ and found by simulation that it has a tolerance of $20 \%$ self-interference effect but decreases $20 \%$ channel capacity. This system uses a full-duplex single channel only. MIMO technology is particularly helpful when capacity, data rates, and link reliability increase without utilizing extra bandwidth or transmission power. Nishizaki et al. [11] observed that the sum capacity when the base station is placed at the wall is larger than one when the base station is placed in massive MIMO because the block SNR maximization and the block diagonalization are introduced in MIMO. Buzzi et al. [12] detected achievable spectral efficiency and energy efficiency for MIMO-based 5G communication system by employing time-domain equalization and advanced equalization techniques. However, it is severely degraded when transmit power falls below $0 \mathrm{~dB}$. Hong et al. [13] obtained optimal detection performance for multiuser MIMO due to exploiting unequal channel reliabilities by employing weighted minimum distance decoding. Loyka et al. [14] learned about the MIMO channel correlation model by scattering environment rank. The assumed factorization of propagation coefficient and physics of radio wave propagation.

Silva et al. [15] analyzed relay-assisted MIMO and obtained a signal-to-interference plus noise ratio for scenarios. To maximize the sum rate and reduce interference below a certain threshold level, an amplify, and forwarding (AF) two- 
way relay is selected. The interference constraints for the primary user are done based on optimal power allocation. The relay also improved energy efficiency. The outage, the sum rate, and the energy efficiency of the network are found to be dependent on relay selection which will increase energy efficiency. A cell-free MIMO system discussed by Ngo et al. [16] has access points that perform conjugate beamforming and employ a pilot assignment algorithm to reduce pilot contamination. The performance of this system in shadow fading gets improved by this algorithm. Solodky et al. [17] proposed a single intra coding scheme for CDMA over automotive MIMO RADAR using the TanSec Frequency Modulation (TSFM) and found by simulation that Frequency-based TSFM outperforms other coding families for crosscorrelation properties. Ravaika et al. [18] implemented OFDM over MIMO and equalizer using LMS algorithm to counteract effects due to fading and interference. Reconfigurable Intelligent Surface (RIS) assisted MIMO-OFDM systems are being significantly used in wireless communications today, which has resulted in improved Mean Square Error (MSE) and Bit Error Rate (BER). Almohammedi et al. [19] derived a new method for estimating Channel State Information (CSI) and found that MIMO exhibited better results. From this literature survey, it is required to find a better technique for improving the performance of the MIMO OFDM system.

\section{5- Problem Statement and Research Methodology}

Co-channel Interference is a threat posed to the spectrum. In such high traffic and mobility situations, problems such as loss of orthogonality occur for MIMO-OFDM systems. The effect of this interference should be reduced and its performance is studied in terms of BER concerning SNR. Two signal processing techniques are being implemented here in this paper to reduce the effect of interference. The figure $4 \mathrm{~b}$ shows how this research is carried out.

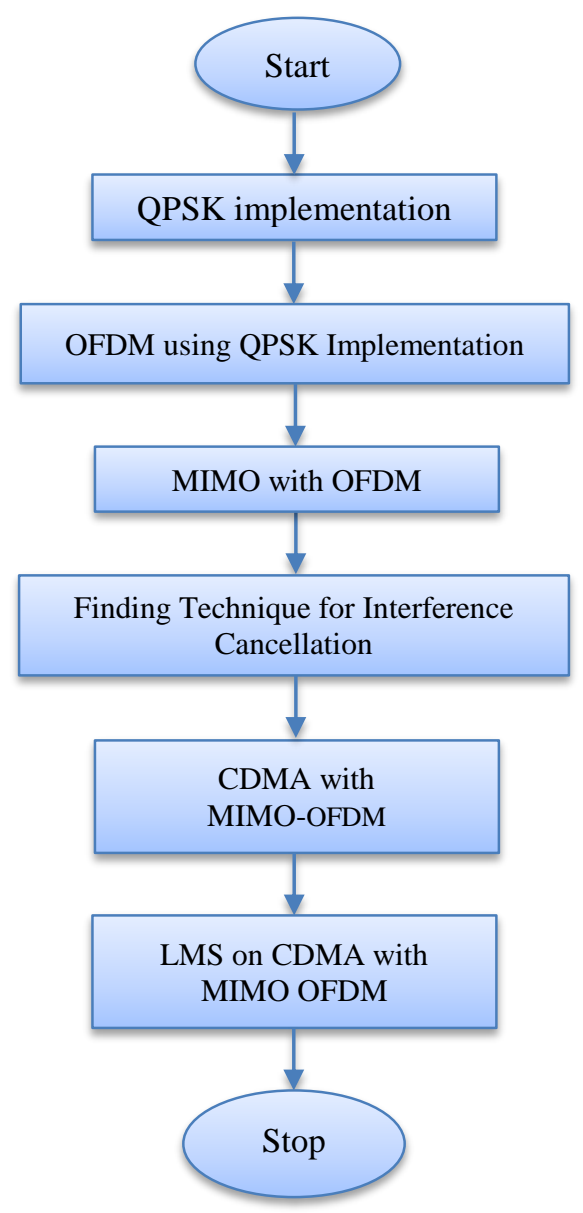

Figure 4. b) Flow chart of the research methodology.

\section{6- The proposed Techniques for the Interference Cancellation}

The first technique is to introduce CDMA in the MIMO-OFDM system, and the second technique is to introduce an LMS filter in a CDMA-MIMO-OFDM system. The performance of both systems is studied and compared [20]. The parameters assumed for simulation are Signal power noise power, pseudo noise sequence length. The range of values considered for studies is within the same limit as other authors. The limitation of the simulation is that it is done for four receivers and numbers of delayed paths. The MIMO -OFDM system with QPSK modulation is a reference system implemented along with the proposed technique due to random noise at the channel. 


\section{6-1- Technique 1-Inclusion of CDMA in MIMO-OFDM Systems}

In modern-day communication systems, bandwidth is a restricted resource. Code Division Multiple Access (CDMA) helps in maximizing its usage. There are two types of CDMA - Direct Sequence and Frequency Hopping techniques. Here, the direct sequence (DS) CDMA technique is implemented.

\section{6-2-DS-CDMA}

Some advantages of CDMA include, complete usage of the available spectrum by every channel, and signals can be encrypted with Pseudo-random (PN) sequences and sent on a wide band of frequencies. The PN sequence is simply multiplied with the random integer generator and given as input to the whole system as seen in Figure 5.

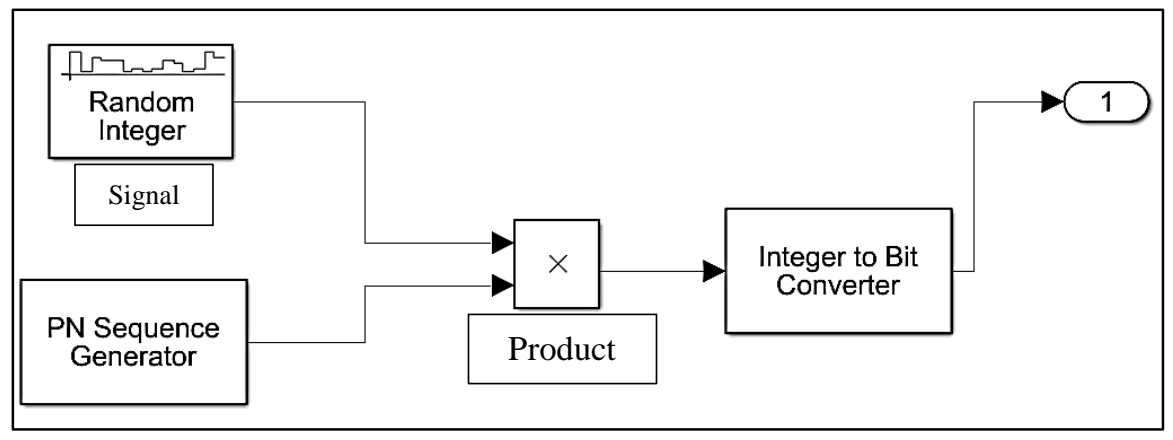

Figure 5. Data source for the CDMA system.

Multiple signals can occupy a single transmission channel in this multiplexing technique, thus improving the use of accessible bandwidth. Unlike TDMA or FDMA, a user in CDMA can access the entire spectrum bandwidth for a complete cycle or duration of time. This is possible only by the use of different codes for each user so that there is no cross-talk or clash between each of them and they can be differentiated. Spreading codes like PN sequences/ Walsh codes come into action while performing the above process. The channel capacity of the spread spectrum is given by the Shannon-Hartley capacity theorem in the following Equation 13.

$C=B * \log _{2}(1+S N R)$

Here, Channel capacity is represented in bits per second (bps). It can also be referred to as the maximum data rate for each bit-error rate (BER). Signal to Noise Ratio can be calculated in $\mathrm{dB}$ and B refers to the required bandwidth in $\mathrm{Hz}$. An added advantage is that due to the wide bandwidth of the spread-spectrum signals, they can be transmitted at a lower power spectral density, which is comparatively lesser than that of the noise power spectral density

In Direct Sequence Spread Spectrum, the users are assigned a particular spreading code. Spreading codes are categorized into two - orthogonal and non-orthogonal. Demodulation of Direct Sequence signals can be done by despreading or a process known as reverse diffusion. The spread spectrum signal is obtained by multiplying the code used in transmission and the reverse diffusion process, the code is further multiplied with the modulated signal. PN sequences are generated by the linear binary feedback shift register. The main reason for using PN sequences here is that it can retain the same transmitter sequence at the receiver side along with the same properties. The probability of error for users in a CDMA system with PN sequences is given by Equation 14 [21].

$P_{e}=2^{-(n-1)} \sum_{B=0}^{n-1}\left(\begin{array}{c}n-1 \\ B\end{array}\right) P_{e \mid B}$

where $\mathrm{n}$ represents the number of classes of PN sequences, $B$ is given as the number of chip boundaries with chip-value transitions and, $P_{e \mid B}$, the conditional probability is given by Equation 15 [21];

$P_{e \mid B}=\frac{1}{2}-\frac{n}{\sqrt{ } 2 \pi} \int_{0}^{\infty} \Phi_{I \mid B}(\omega) \exp \left\{-\frac{1}{2} \omega^{2} n^{2}\right\} d \omega$

Here, the value of B gives an estimate of the average BER experienced by user and $\Phi_{I \mid B}(\omega)$ represents the conditional CF. $P_{e}$ experienced in a CDMA system with QPSK modulation scheme is given in Equation 16 [22];

$P_{e}=\frac{1}{2}\left(P_{e}^{I}+P_{e}^{Q}\right)$

where, $P_{e}^{I}$ and $P_{e}^{Q}$ represent in-phase and quadrature components of the QPSK signal. 


\section{6-3- Technique 2 - Inclusion of LMS Filter in CDMA-MIMO-OFDM Systems}

The LMS algorithm followed for interference cancellation is given below. This technique is preferred because its computational complexity is very low. $\mathrm{y}[\mathrm{n}]$ is the output signal and $\mathrm{x}[\mathrm{n}]$ is the input signal in Equation 17 and is computed by using the standard FIR formulation;

$y[n]=b[0] * x[n]+b[1] * x[n-1]+b[2] * x[n-2]+\cdots+b[$ numTaps -1$] * x[$ numTaps -1$]$

The error signal in Equation 18 calculates the difference between the desired reference signal d[n] and the filter output;

$e[n]=d[n]-y[n]$

After computation of each iteration of the error signal, the filter coefficients $b[\mathrm{k}]$ are updated as shown in. Equation 19.

$b[k]=b[k]+e[n] * m u * x[n-k]$, for $k=0,1, \ldots$, numTaps -1

where mu is the step size and controls the rate of coefficient convergence. Average Probability of error for LMS induced system becomes, as shown in Equation 20;

$P_{\alpha}=\frac{1}{N B} \sum_{l=1}^{B} \sum_{n=1}^{N} P(n Q, l)$

Averaging finally gives Equation 21;

$P=\sum_{\alpha=1}^{2} p(\alpha) P_{\alpha}$

where $p(\alpha)$ is a prior distribution of one and zero in binary form. Although, it can be given as Equation 22 most of the times $[23,24]$.

$P=0.5\left(P_{1}+P_{2}\right)$

\section{7- Proposed Technique Simulation Using MATLAB-SIMULINK}

\section{7-1- Proposed Technique 1: Inclusion of CDMA with MIMO-OFDM System}

The entire CDMA-MIMO-OFDM model with two receiver antennas is given in Figures 6 and 7. The data source block has an in-built block with CDMA technology. The IQ Mapper block modulates the incoming signal by QPSK modulation. The modulated signal undergoes OFDM and space-time block encoding. Additionally, co-channel interference is introduced to the signal path. The signal is transmitted through AWGN and Rayleigh/ Rician channels. At the receiver end, the signal is decoded concerning STBC and goes through OFDM demodulation. The IQ de-mapper block demodulates the QPSK signal. Finally, the Data Sink block generates transmitted data from the demodulated signal. The BER is calculated and displayed by the Error Rate Calculation block.

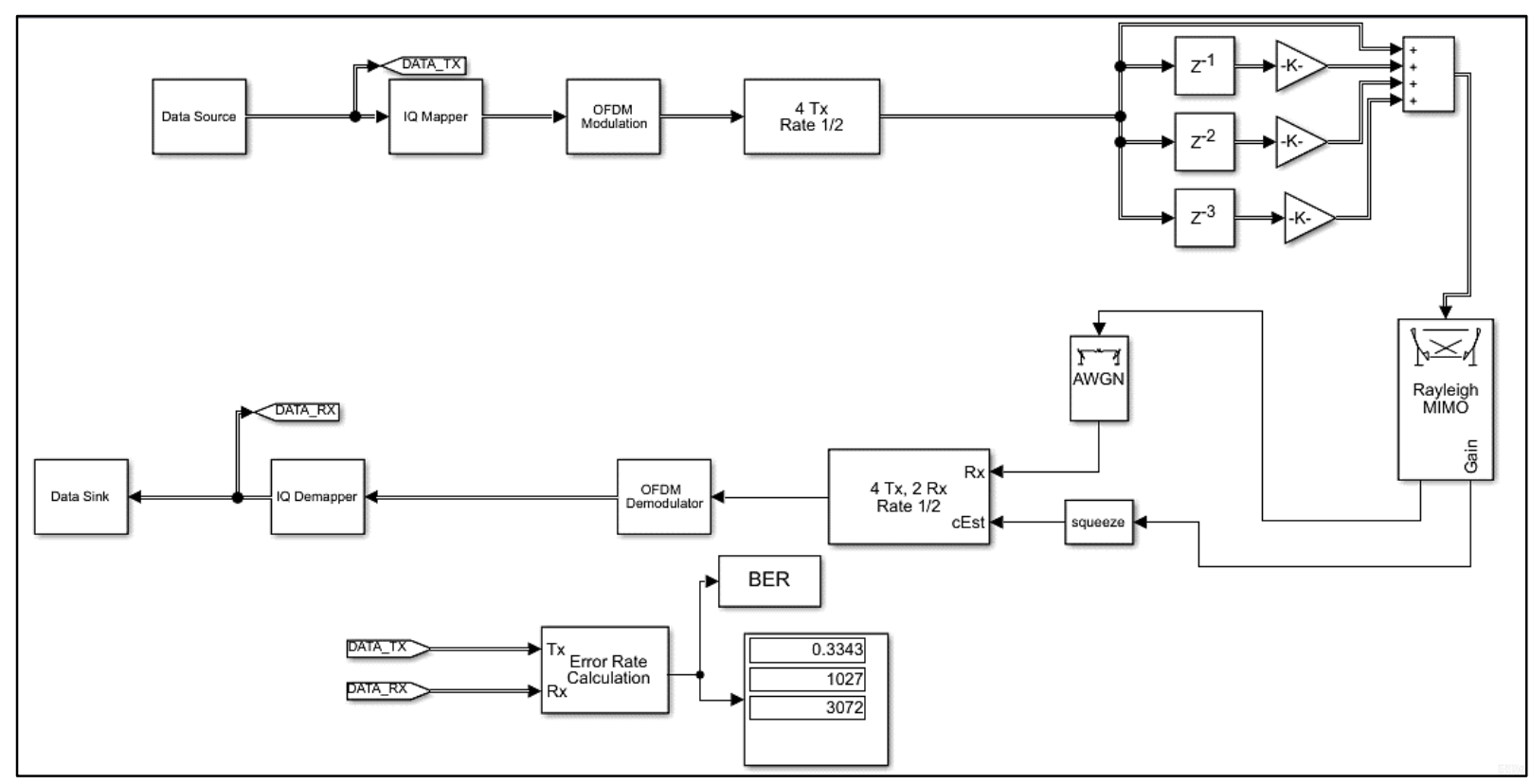

Figure 6. CDMA-MIMO-OFDM Model in Rayleigh Channel. 


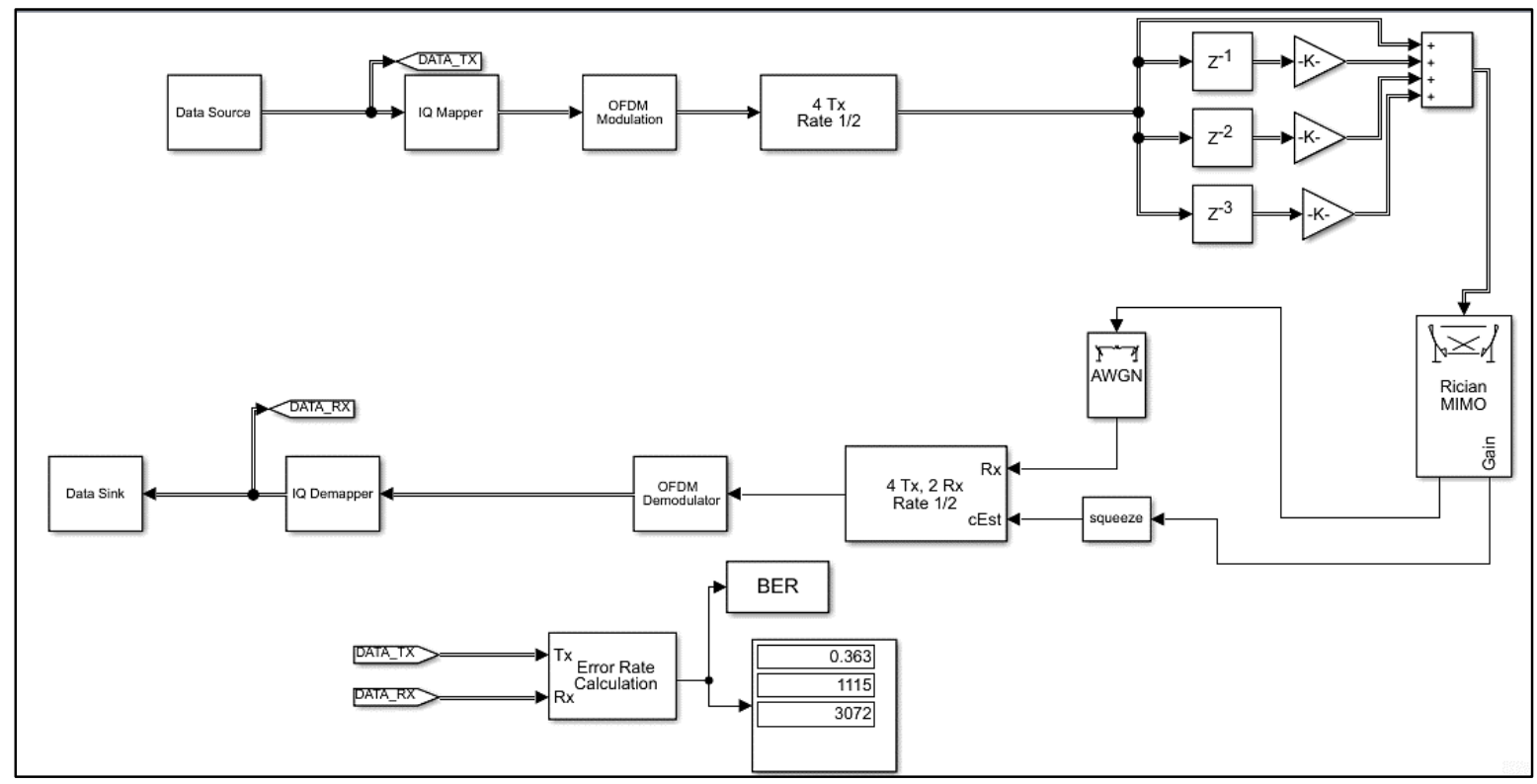

Figure 7. CDMA-MIMO-OFDM Model in Rician Channel.

\section{7-2- Proposed Technique 2: Inclusion of LMS filter with CDMA-MIMO-OFDM system}

The LMS filter block in Figure 8 has been included in that model to fight the effect of interference and help in interference cancellation. The corrupted signal that has travelled through the noise channels is given as input to this block and the original OFDM signal is given as the desired signal so that the filter can mimic its performance. The configuration can be changed for $4 \times 1$ systems too [25].

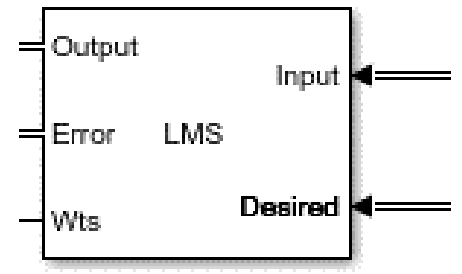

Figure 8. LMS Filter Block used in the model

Figures 9 and 10 represents the block diagram for the LMS-MIMO-OFDM 4×1 system for both Rayleigh and Rician channels respectively.

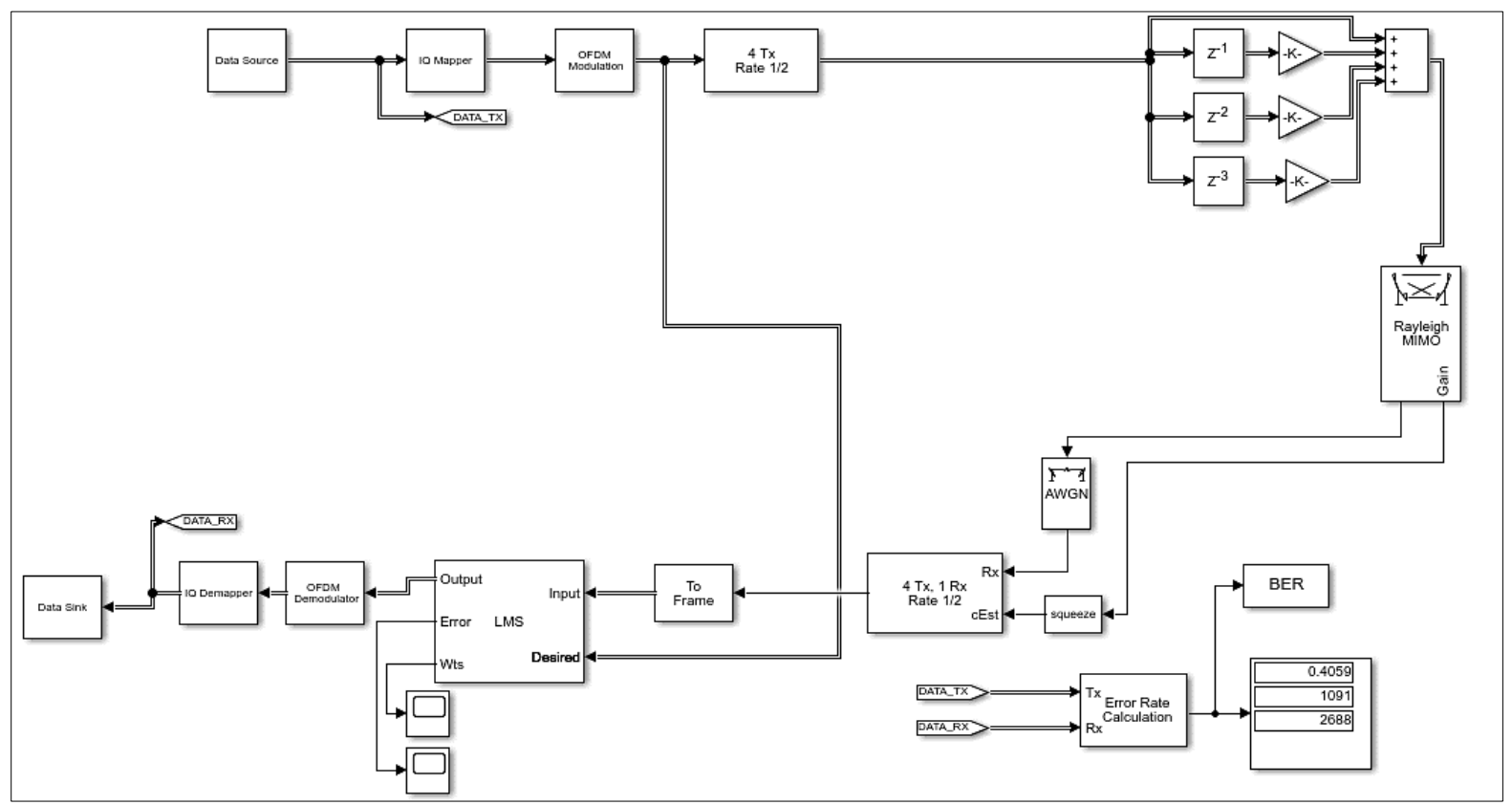

Figure 9. 4×1 LMS-MIMO-OFDM Model in Rician Channel. 


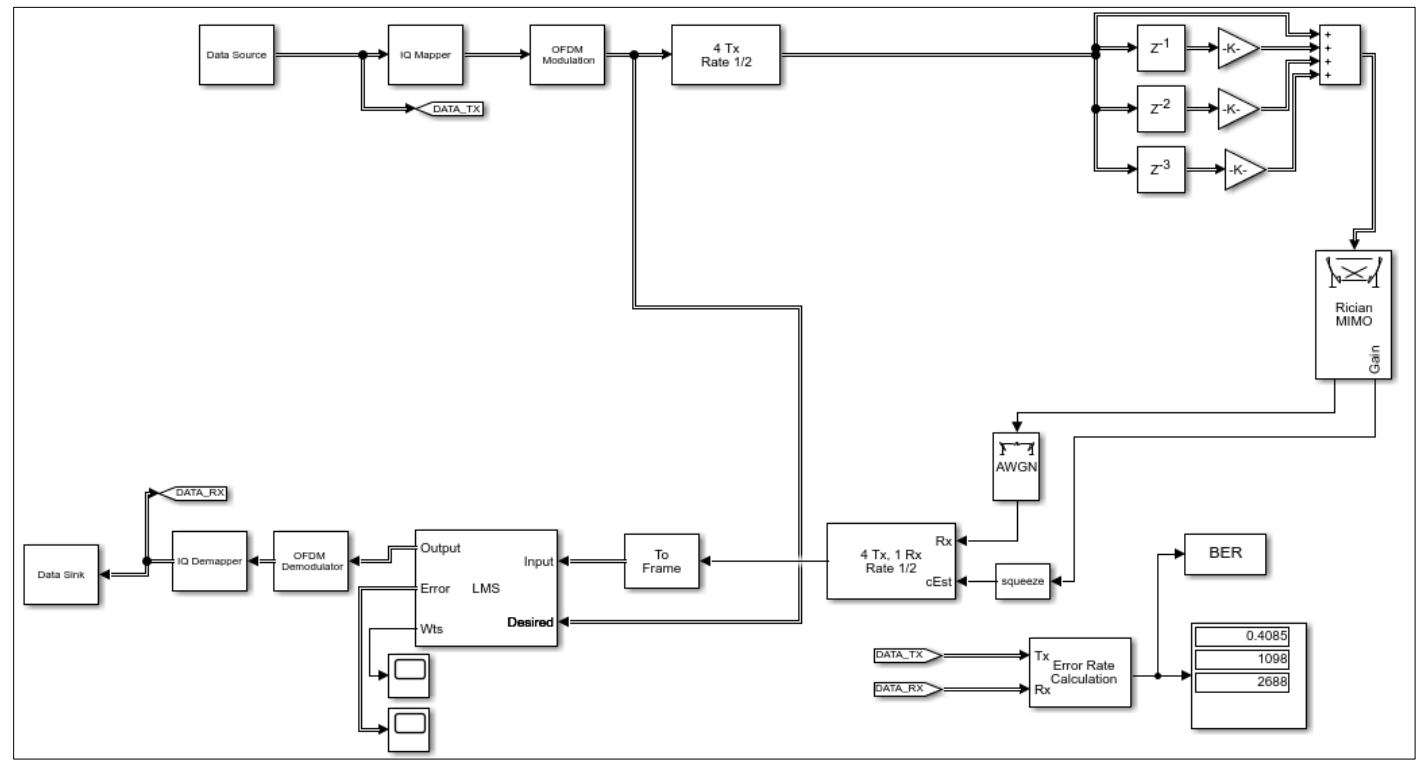

Figure 10. 4×1 LMS-MIMO-OFDM Model in Rician Channel.

Figures 11 and 12 represents the block diagram for the LMS-MIMO-OFDM $4 \times 4$ system for both Rayleigh and Rician channels respectively.

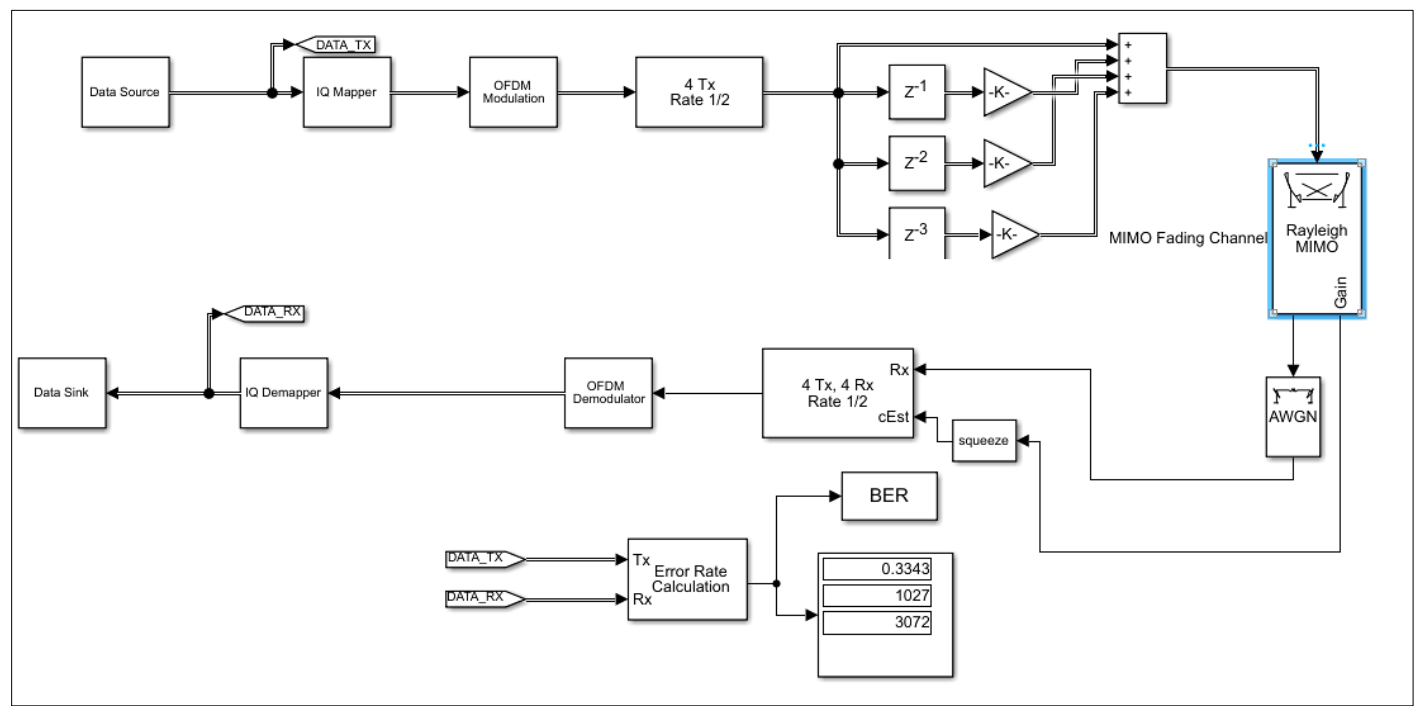

Figure 11. 4×4 LMS-MIMO-OFDM Model in Rayleigh Channel.

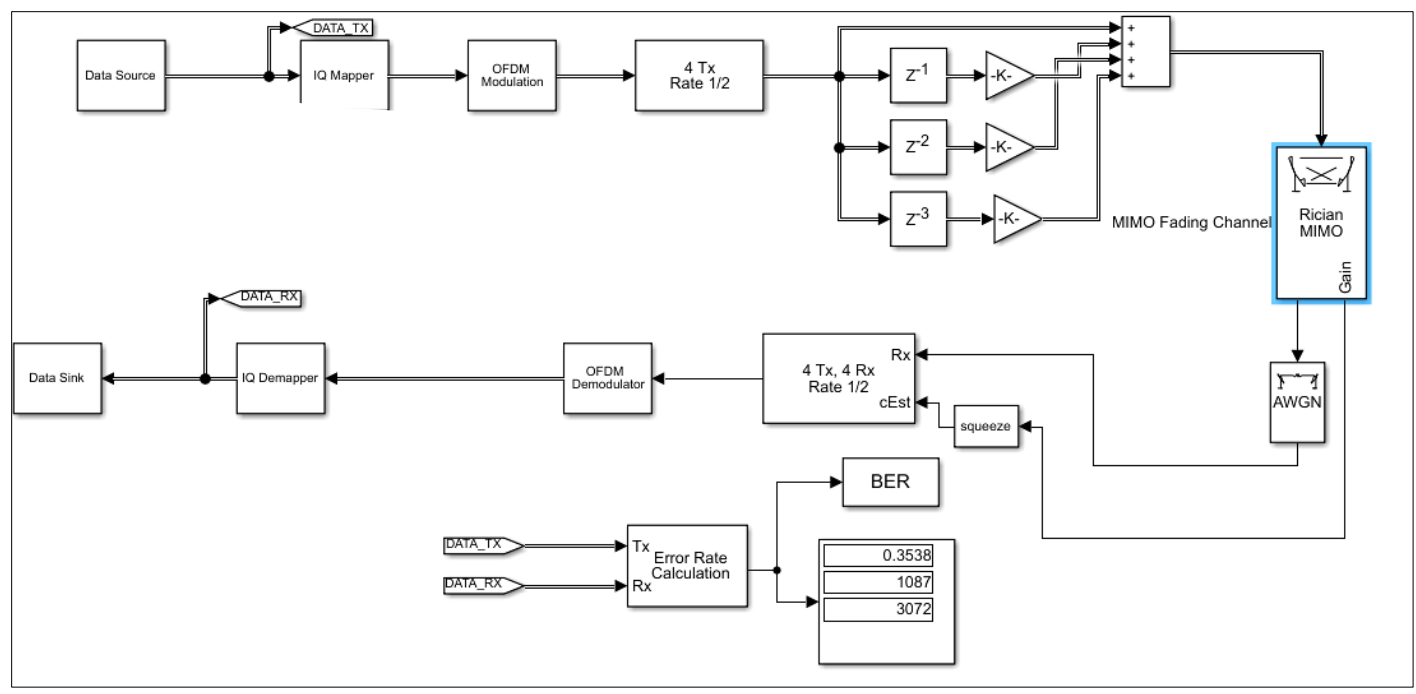

Figure 12. 4×4 LMS-MIMO-OFDM Model in Rayleigh Channel. 


\section{8- Results and Discussions}

\section{8-1- Simulation Results - Technique 1 (CDMA-MIMO-OFDM)}

BER Performance of CDMA-MIMO-OFDM systems in Rayleigh channel is plotted as shown in Figure 13 and for Rician channel in Figure 14 correspondingly [26-30]. With an increase in Eb/N0, the BER decreases. From Table 1, when Eb/N0 is $15 \mathrm{~dB}$, BER is 0.3727 for $4 \times 4$ systems in Rayleigh channel. From Table 2, when Eb/N0 is $15 \mathrm{~dB}$, BER is 0.3429 for $4 \times 4$ systems in Rayleigh channel. It is seen that the $4 \times 2$ system provides slightly better performance when compared to the $4 \times 1$ system and the $4 \times 4$ system performs even better than the $4 \times 2$ system. Even though $4 \times 4$ has the possibility of more interference, it performs equally as $4 \times 1$ or $4 \times 2$. The plausible reason for this is due to the effect of CDMA. The values corresponding to $4 \times 4$ systems in Figures 18 and 19 are seen below in Tables 1 and 2 respectively.

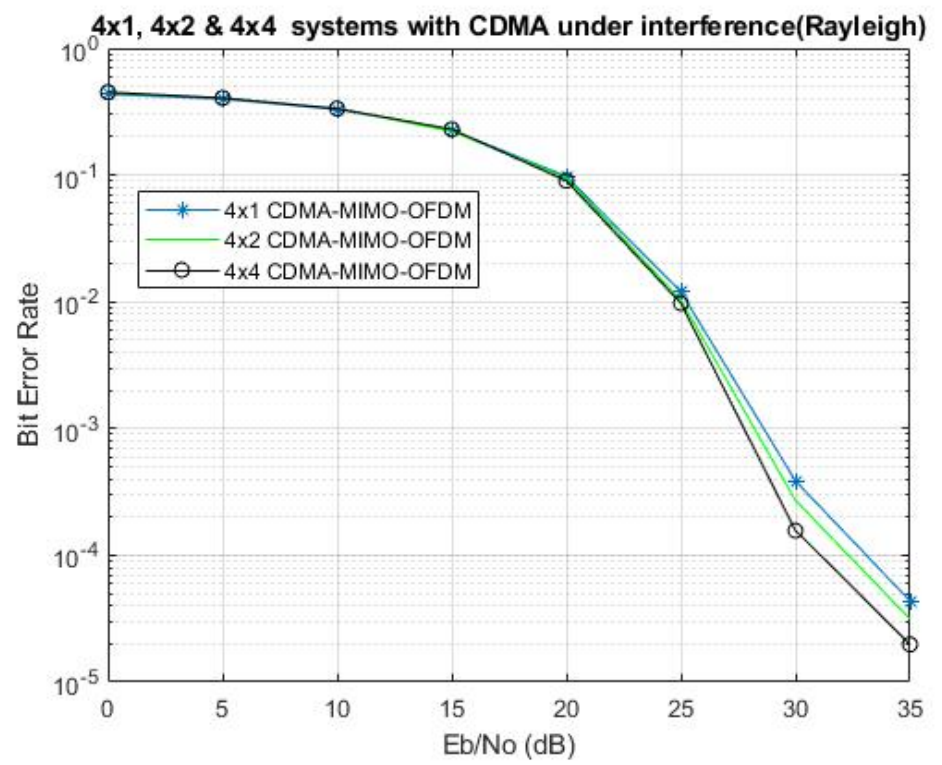

Figure 13. SNR vs. BER plot in Rayleigh channel for CDMA-MIMO-OFDM model.

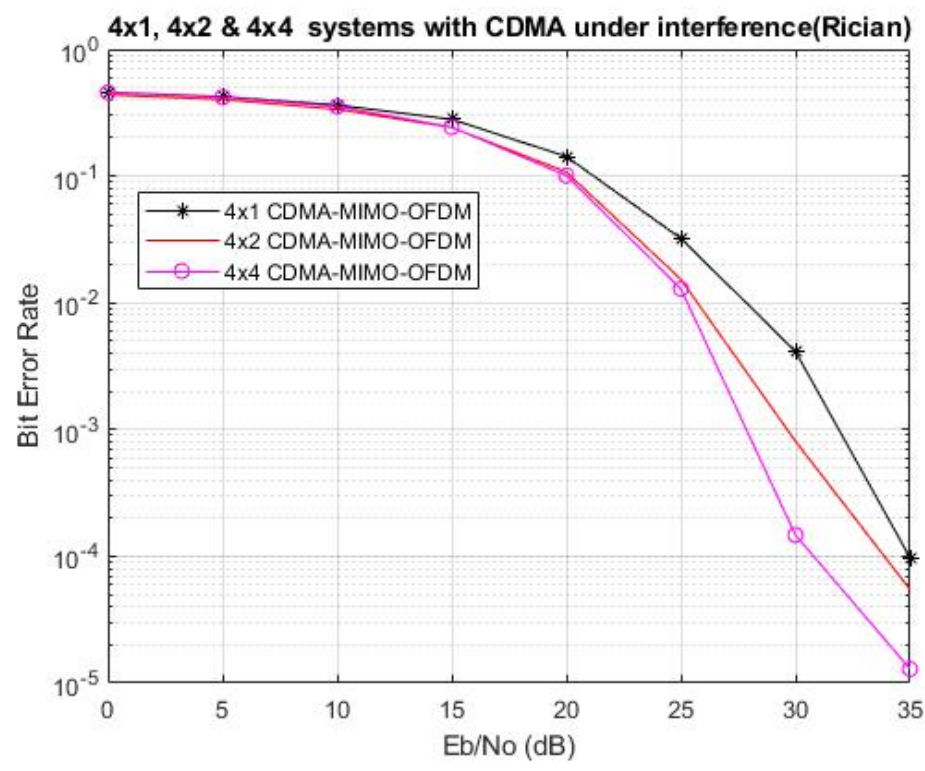

Figure 14. SNR vs. BER plot in Rician channel for CDMA-MIMO-OFDM model.

Table 1. BER Values corresponding to $5 \mathrm{~dB}, 15 \mathrm{~dB}, 25 \mathrm{~dB}$ and $35 \mathrm{~dB}$. SNR in Rayleigh channel.

\begin{tabular}{ccccc}
\hline Technique/SNR & $\mathbf{5 ~ d B}$ & $\mathbf{1 5} \mathbf{~ d B}$ & $\mathbf{2 5} \mathbf{~ d B}$ & $\mathbf{3 5} \mathbf{~ d B}$ \\
\hline MIMO-OFDM (reference model) & 0.5066 & 0.3727 & 0.1029 & $6.042 \mathrm{e}-04$ \\
With CDMA & 0.5035 & 0.2946 & 0.01329 & $9.127 \mathrm{e}-05$ \\
With CDMA and LMS & 0.4177 & 0.2423 & 0.01290 & $3.423 \mathrm{e}-05$ \\
\hline
\end{tabular}


Table 2. BER Values corresponding to $5 \mathrm{~dB}, 15 \mathrm{~dB}, 25 \mathrm{~dB}$ and $35 \mathrm{~dB}$. SNR in Rician channel.

\begin{tabular}{ccccc}
\hline Technique/SNR & $\mathbf{5 ~ d B}$ & $\mathbf{1 5} \mathbf{~ d B}$ & $\mathbf{2 5} \mathbf{~ d B}$ & $\mathbf{3 5} \mathbf{~ d B}$ \\
\hline MIMO-OFDM (reference model) & 0.5182 & 0.3429 & 0.11287 & $7.419 \mathrm{e}-04$ \\
With CDMA for interference cancellation & 0.4891 & 0.3058 & 0.03103 & $9.289 \mathrm{e}-05$ \\
With CDMA and LMS for interference cancellation & 0.4066 & 0.2216 & 0.01029 & $5.558 \mathrm{e}-05$ \\
\hline
\end{tabular}

\section{8-2 Simulation Results - Technique 2 (CDMA-MIMO-OFDM with LMS filter)}

BER of CDMA-MIMO-OFDM systems with LMS filter is plotted as shown in Figure 15 for a $4 \times 1$ system, Figure 16 for a $4 \times 2$ system, and Figure 17 for a $4 \times 4$ system respectively. From the plots in Figures 15 to 20 , it is understandable that the performance of both the MIMO systems under Rayleigh and Rician channels have improved after interference cancellation due to the implementation of the filter with the LMS algorithm. The BER is appreciably reduced, thus serving the purpose of interference cancellation. It proves to be better than CDMA-MIMO-OFDM. The values corresponding to Figures 19 and 20 are also seen below in Tables 1 and 2 respectively. The contribution of the research is to reduce the effect of interference. When the system is taken for implementation practically, the non-availability of the delayed path becomes its challenge and difficulty. Figures 15 and 16 show the improvement of $4 \times 1$ MIMO-OFDM systems in the presence of CDMA and LMS filter in Rayleigh and Rician channels.

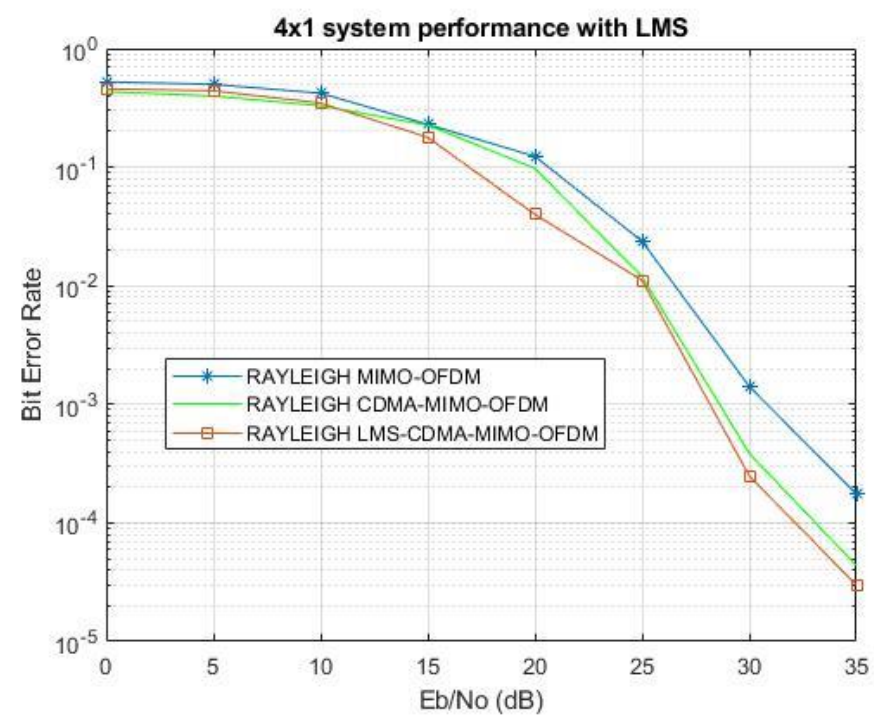

Figure 15. SNR vs. BER plot for $4 \times 1$ LMS-MIMO-OFDM model in Rayleigh channel.

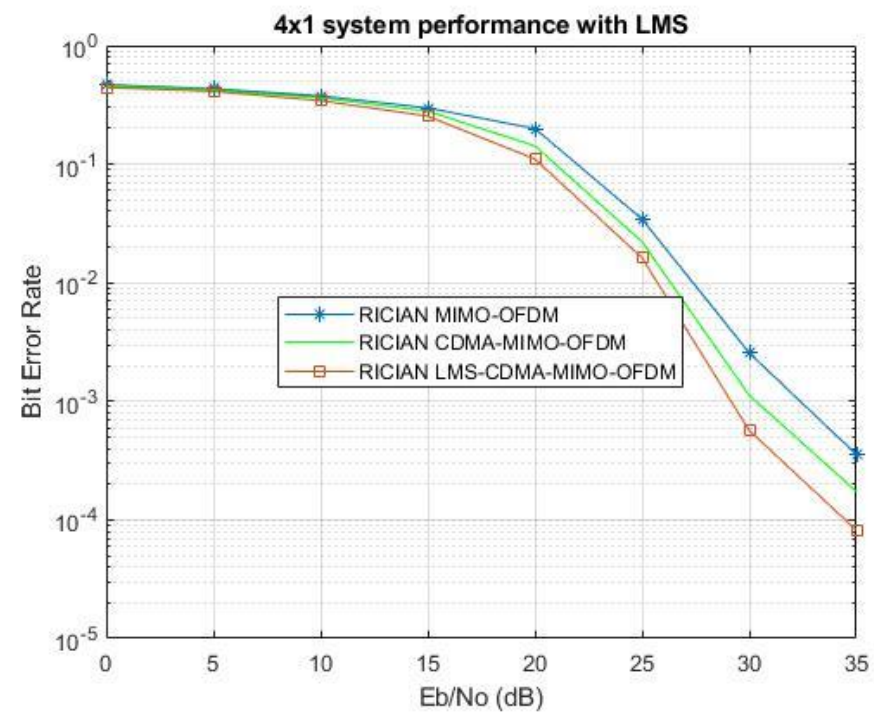

Figure 16. SNR vs. BER plot for 4×1 LMS-MIMO-OFDM model in Rician channel.

Figures $17 \& 18$ show the improvement of $4 \times 2$ MIMO-OFDM systems in the presence of CDMA and LMS filter in Rayleigh and Rician channels. 


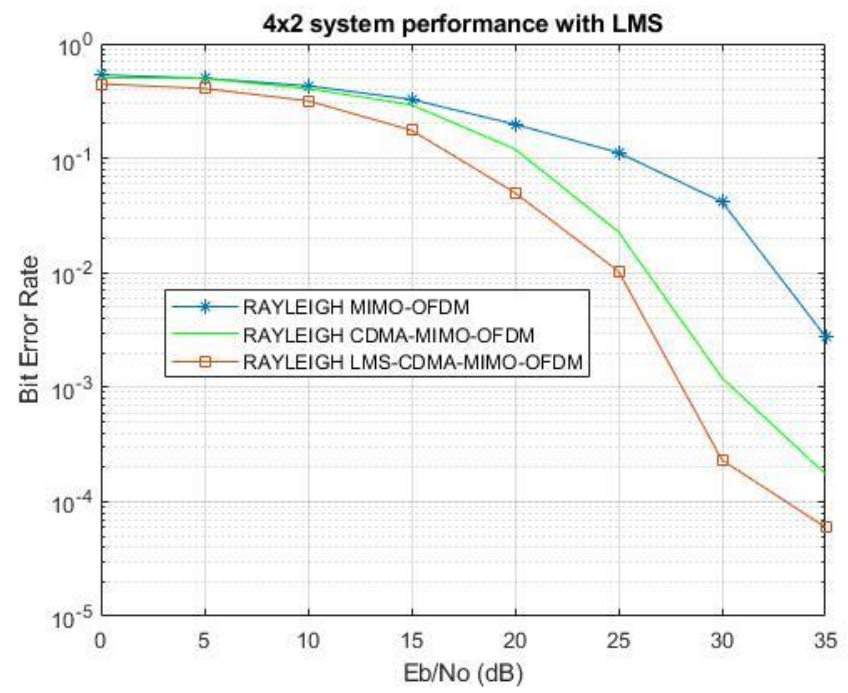

Figure 17. SNR vs. BER plot for 4×2 LMS-MIMO-OFDM model in Rayleigh channel.

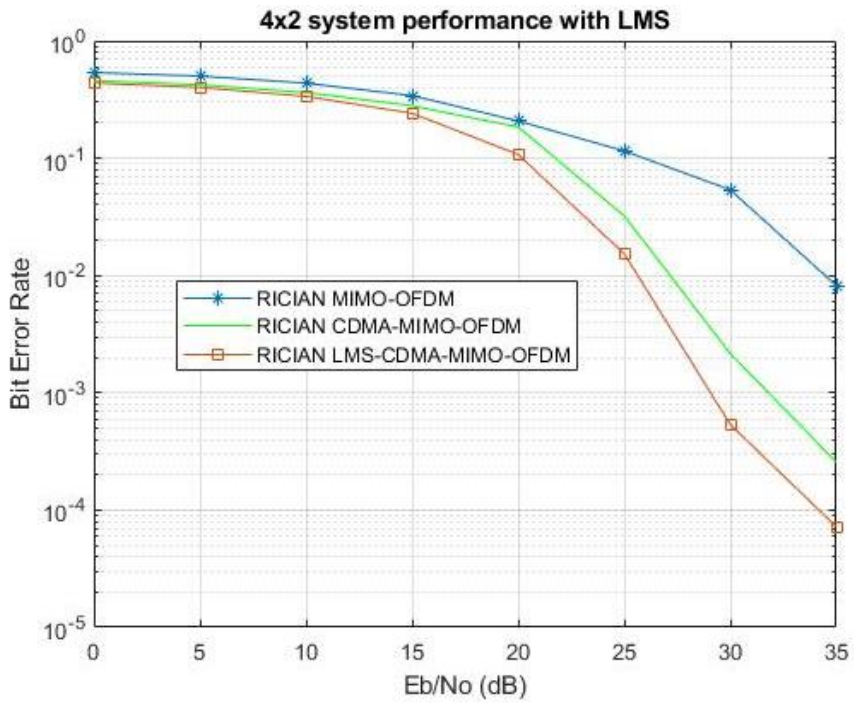

Figure 18. SNR vs. BER plot for $4 \times 2$ LMS-MIMO-OFDM model in Rician channel.

Figures 19 and 20 show the improvement of 4×4 MIMO-OFDM systems in the presence of CDMA and LMS filter in Rayleigh and Rician channels.

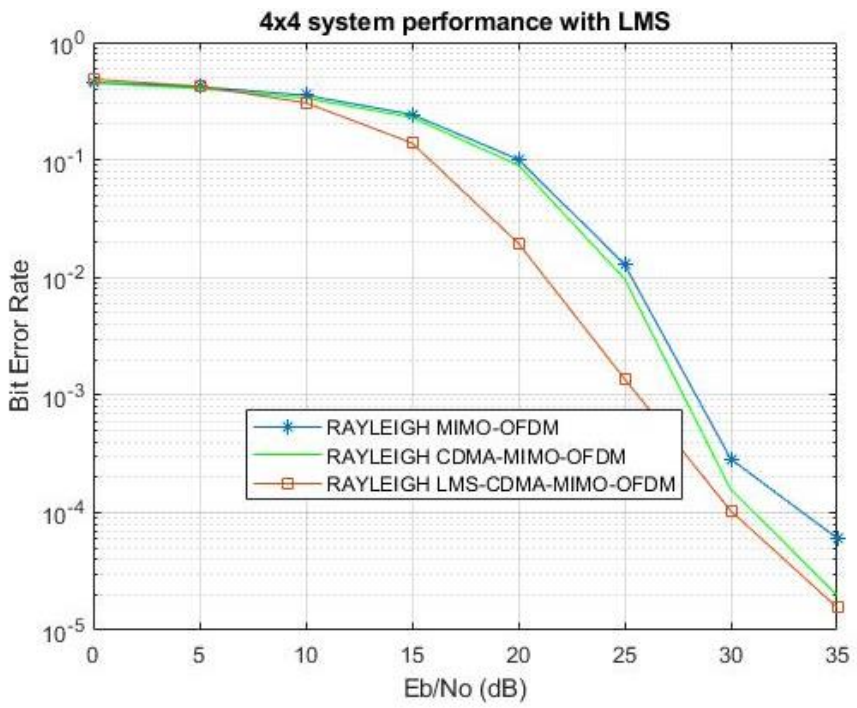

Figure 19. SNR vs. BER plot for 4×4 LMS-MIMO-OFDM model in Rayleigh Channel. 


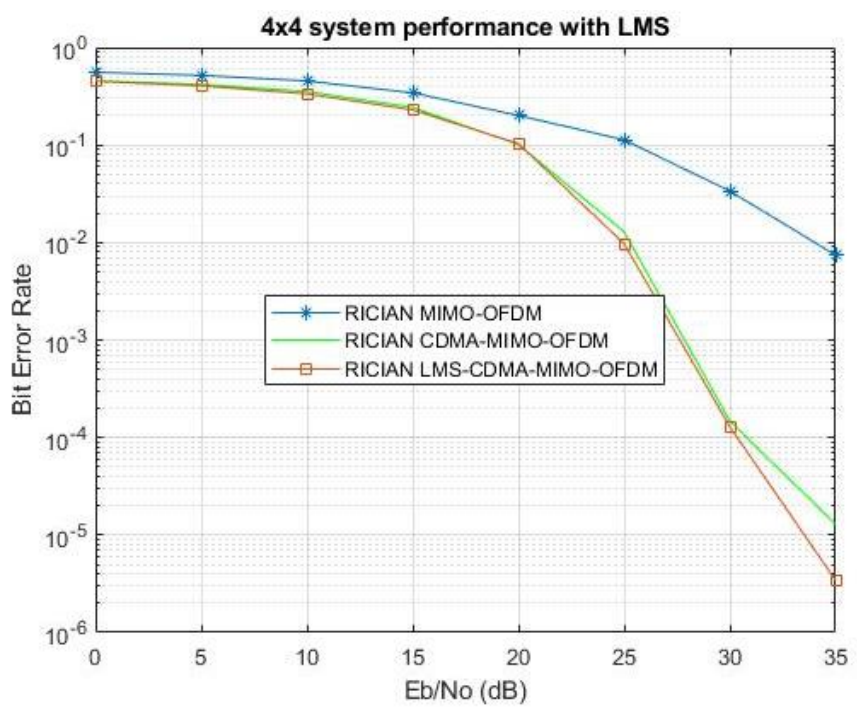

Figure 20. SNR vs. BER plot for 4×4 LMS-MIMO-OFDM model in Rician Channel.

\section{9- Conclusion}

MIMO-OFDM has been seen to be such an important part of wireless systems and thus, has been studied in this paper simulation. MIMO technology is implemented in the standard using three different techniques: antenna selection, space-time coding and possibly beamforming. MIMO is also planned to be used in Mobile radio telephone. Moreover, to fully support cellular environments, MIMO wireless communications architectures and processing techniques are applied to MIMO radar. In cognitive radio networks, the radio spectrum resources utilization is improved by allowing a greater number of users. MIMO can build wireless network such as WLAN having standard WiFi 802.11n, Muni Wireless, MAN with WiMAX 802.16e standard, 5G Mobile networks, RFID communication and Digital Home. The performance of this MIMO is getting affected by co channel interference. It is required to have proper solution for this issue.

This research article aims for the same and has deciphered the following results. In this paper, the performance of co-channel interference cancellation technique for MIMO-OFDM systems in the presence of CDMA and interference has been studied in terms of the Signal-to-noise ratio vs. Bit-error rate (BER) comparison. Co-channel Interference results due to the delay introduced to the proposed system model which degrades the performance by providing a loss of bits in the receiver side. Furthermore, two interference cancellation techniques were also implemented and were proved to be effective in reducing interference for MIMO-OFDM systems. CDMA and LMS filter have been implemented as interference cancellation techniques. A CDMA-MIMO-OFDM system with an LMS filter has been found to show better results in terms of reducing the bit error rate. Only Simulink MATLAB simulation is done. The results are sensitive to signal power and noise power and interference power.Going along the lines of future scope, this model is tested with only QPSK modulation and can also be extended to other modulation schemes such as GMSK. Appropriate interference cancellation filtering techniques are to be researched to combat the interference issue in this model.

\section{0- Declarations}

\section{0-1- Author Contributions}

Conceptualization, A.R.; methodology, A.R. and V.R.; software, V.R.; validation, A.R. and V.R.; formal analysis, A.R. and V.R.; investigation, A.R. and V.R.; resources, A.R. and V.R.; data curation, A.R. and V.R.; writing-original draft preparation, A.R. and V.R.; writing-review and editing, A.R. and V.R.; visualization, A.R. and V.R.; supervision, A.R. and V.R.; project administration, A.R.; funding acquisition, A.R. All authors have read and agreed to the published version of the manuscript.

\section{0-2- Data Availability Statement}

Data sharing is not applicable to this article.

\section{0-3- Funding}

The authors received no financial support for the research, authorship, and/or publication of this article. 


\section{0-4- Acknowledgements}

The authors would like to thank the Director, Birla Institute of Technology and Science Pilani, Dubai campus. This research work was supported by the communication laboratory at Birla Institute of Technology And Science Pilani, Dubai campus.

\section{0-5- Conflicts of Interest}

The authors declare that there is no conflict of interests regarding the publication of this manuscript. In addition, the ethical issues, including plagiarism, informed consent, misconduct, data fabrication and/or falsification, double publication and/or submission, and redundancies have been completely observed by the authors.

\section{1- References}

[1] Dugad, Payal, and Ashok Khedkar. "Performance of OFDM System for AWGN, Rayleigh and Rician Channel." International Journal of Innovative Research in Computer and Communication Engineering 5, no. 7 (2017).

[2] Tse, David, and Viswanath Pramod. "Fundamentals of Wireless Communication." Fundamentals of Wireless Communication, (2005). doi:10.1017/CBO9780511807213.

[3] Omijeh, B O, and Ken Saro-wiwa. "Bit Error Rate and Signal to Noise Ratio Performance Evaluation of OFDM System with QPSK and QAM M-Array Modulation Scheme in Rayleigh , Rician and AWGN Channel Using MATLAB / Simulink." Innovative Systems Design and Engineering 8, no. 4 (2017).

[4] Awon, Nuzhat Tasneem, Md. Ashraful Islam, Md. Mizanur Rahman, and A. Z. M. Touhidul Islam. "Effect of AWGN \& Fading (Raleigh \& Rician) Channels on BER Performance of a WiMAX Communication System.”. International Journal of Computer Science and Information Security 10, no. 8 (2012).

[5] Ogale, Archana, Shubhangi Chaoudhary, and A. J. Patil. "Performance Evaluation of MIMO-OFDM System Using Matlab® Simulink with Real Time Image Input.” In IFIP International Conference on Wireless and Optical Communications Networks, WOCN, 1-5, 2013. doi:10.1109/WOCN.2013.6616176.

[6] Sinha, Nirmalendu Bikas, R. Bera, S. Manna, Shashank Gupta, and M. Mitra. "Hybrid Technology for next Generation Broad Band Mobile Radio Communications." 2009 IFIP International Conference on Wireless and Optical Communications Networks, WOCN 2009, 2009. doi:10.1109/WOCN.2009.5010504.

[7] Shah, Yousaf Ali. "Performance Analysis of Multiple Antenna Techniques in Wimax." Technology, (2010).

[8] Space-time block code, Wikipedia. Available online: https://en.wikipedia.org/wiki/Space\%E2\%80\%93time_block _code\#Alamouti's_code (accessed on May 2021).

[9] Ogale, Archana, Shubhangi Chaoudhary, and A. J. Patil. "Performance Evaluation of MIMO-OFDM System Using Matlab® Simulink with Real Time Image Input." In IFIP International Conference on Wireless and Optical Communications Networks, WOCN, (2013):1-5. doi:10.1109/WOCN.2013.6616176.

[10] Larashati, Giashinta, Rina Pudji Astuti, and Bambang Setia Nugroho. "Modeling of Massive MIMO Transceiver Antenna for Full-Duplex Single-Channel System (in Case of Self Interference Effect).” In Proceedings - International Conference on Signals and Systems, ICSigSys 2017, 1-5, (2017). doi:10.1109/ICSIGSYS.2017.7967021.

[11] Nishizaki, Shun, Toshihiko Nishimura, Takeo Ohgane, and Yasutaka Ogawa. "Performance Comparison of Full-Dimension MIMO Systems with Different Base Station Places.” 2017 International Workshop on Antenna Technology: Small Antennas, Innovative Structures, and Applications, IWAT 2017, (2017):335-38. doi:10.1109/IWAT.2017.7915394.

[12] Buzzi, Stefano, Carmen D’Andrea, Tommaso Foggi, Alessandro Ugolini, and Giulio Colavolpe. "Single-Carrier Modulation versus OFDM for Millimeter-Wave Wireless MIMO.” IEEE Transactions on Communications 66, no. 3 (2018): $1335-48$. doi:10.1109/TCOMM.2017.2771334.

[13] Hong, Song Nam, Seonho Kim, and Namyoon Lee. "A Weighted Minimum Distance Decoding for Uplink Multiuser MIMO Systems with Low-Resolution ADCs.” IEEE Transactions on Communications 66, no. 5 (2018): 1912-24. doi:10.1109/TCOMM.2017.2787565.

[14] Loyka, Sergey, and Mahdi Khojastehnia. "Comments on 'On Favorable Propagation in Massive MIMO Systems and Different Antenna Configurations.” IEEE Access 7 (2019): 185369-72. doi:10.1109/ACCESS.2019.2960025.

[15] Silva, Shashindra, Masoud Ardakani, and Chintha Tellambura. "Interference Suppression and Energy Efficiency Improvement with Massive MIMO and Relay Selection in Cognitive Two-Way Relay Networks." IEEE Transactions on Green Communications and Networking 4, no. 2 (2020): 326-39. doi:10.1109/TGCN.2020.2965116.

[16] Ngo, Hien Quoc, Alexei Ashikhmin, Hong Yang, Erik G. Larsson, and Thomas L. Marzetta. “Correction to 'Cell-Free Massive MIMO versus Small Cells.” IEEE Transactions on Wireless Communications 19, no. 5 (2020): 3623-24. doi:10.1109/TWC.2020.2974209. 
[17] Solodky, Gaston, Oren Longman, Shahar Villeval, and Igal Bilik. "CDMA-MIMO Radar with the Tansec Waveform." IEEE Transactions on Aerospace and Electronic Systems 57, no. 1 (2021): 76-89. doi:10.1109/TAES.2020.3016876.

[18] Ravalika, Yerpula, and Gihginha Swetha. "Implementation of Non-Linear Adaptive Equalizer for MIMO-OFDM in Wireless Communication." In Proceedings of the 4th International Conference on Electronics, Communication and Aerospace Technology, ICECA 2020, (2020): 532-37. doi:10.1109/ICECA49313.2020.9297435.

[19] Almohammedi, Ali, and Mohamed Deriche. "LMS for Truncate CSI Feedback in Massive MIMO.” In Proceedings - 2019 International Conference on Computing, Electronics and Communications Engineering, ICCECE 2019, (2019): 241-46. doi:10.1109/iCCECE46942.2019.8941771.

[20] Sethy, Prabira Kumar, and Subrata Bhattacharya. "Interference Cancellation in Adaptive Filtering through LMS Algorithm Using TMS320C6713DSK." International Journal of Electronics and Communication Engineering 5, no. 2 (2012): 113-24.

[21] Liu, Xiang, and Lajos Hanzo. “Accurate BER Analysis of QPSK Modulated Asynchronous DS-CDMA Systems Communicating over Rayleigh Channels.” In IEEE Vehicular Technology Conference, 4:1640-44, 2006. doi:10.1109/vetecs.2006.1683124.

[22] Cheng, Julian, and Norman C. Beaulieu. “Accurate DS-CDMA Bit-Error Probability Calculation in Rayleigh Fading.” IEEE Transactions on Wireless Communications 1, no. 1 (2002): 3-14. doi:10.1109/7693.975440.

[23] Glisic, Savo G. "Multilayer LMS Interference Suppression Algorithms for CDMA Wireless Networks.” IEEE Transactions on Communications 48, no. 8 (2000): 1413-23. doi:10.1109/26.864178.

[24] Mayyas, K. "Performance Analysis of the Deficient Length LMS Adaptive Algorithm." IEEE Transactions on Signal Processing 53, no. 8 (2005): 2727-34. doi:10.1109/TSP.2005.850347.

[25] Vasu, Rohit, Abdul Rajak, and Shazia Hassan. "Design and Simulation of Single Ended Double Balanced Rf Gilbert Mixers." ARPN Journal of Engineering and Applied Sciences 15, no. 20 (2020): 2238-43.

[26] Rajak, A R Abdul, Azeem Gafoor Mohammed, and Deep Sehgal. "Design and Analysis of High-Speed Phase Locked Loop in 180 Nm Technology." Intelligent Electrical Systems: A Step towards Smarter Earth. Institute of Engineering \& Management, at S.N Bose National Centre for Basic Sciences Kolkata, (2020). doi:10.1201/9780429355998-26.

[27] Rajesh, Varshini, and A. R.Abdul Rajak. "Channel Estimation for Image Restoration Using OFDM with Various Digital Modulation Schemes.” In Journal of Physics: Conference Series, 1706:13 -14, (2020). doi:10.1088/1742-6596/1706/1/012076.

[28] Sreejith, Shwetha, and Abdul Rajak. "Study on Optimization of Handoff Process Using Fuzzy Logic for Mobile Communication.” In Journal of Physics: Conference Series, 1706:13 -14, (2020). doi:10.1088/1742-6596/1706/1/012161.

[29] Smrithi, M N, and A R Abdul Rajak. "Design and Implementation of FEC for CDMA System." In IOP Conference Series: Materials Science and Engineering, 1045:012038, (2021). doi:10.1088/1757-899x/1045/1/012038.

[30] Smrithi, M N, and A R Abdul Rajak. "Design and Implementation of FEC for CDMA System.” IOP Conference Series: Materials Science and Engineering 1045, no. 1 (February 1, 2021): 012038. doi:10.1088/1757-899x/1045/1/012038. 